$12-1-2011$

\title{
Urolithiasis location and size and the association with microhematuria and stone-related symptoms.
}

\author{
Costas D Lallas \\ Department of Urology, Thomas Jefferson University \\ Xiaolong S Liu \\ Department of Urology, Thomas Jefferson University
}

Allen N Chiura

Department of Urology, Thomas Jefferson University

Akhil K Das

Department of Urology, Thomas Jefferson University

Demetrius H Bagley

Department of Urology, Thomas Jefferson University

Follow this and additional works at: https://jdc.jefferson.edu/urologyfp

Part of the Urology Commons

Let us know how access to this document benefits you

\section{Recommended Citation}

Lallas, Costas D; Liu, Xiaolong S; Chiura, Allen N; Das, Akhil K; and Bagley, Demetrius H, "Urolithiasis location and size and the association with microhematuria and stone-related symptoms." (2011). Department of Urology Faculty Papers. Paper 15.

https://jdc.jefferson.edu/urologyfp/15

This Article is brought to you for free and open access by the Jefferson Digital Commons. The Jefferson Digital Commons is a service of Thomas Jefferson University's Center for Teaching and Learning (CTL). The Commons is a showcase for Jefferson books and journals, peer-reviewed scholarly publications, unique historical collections from the University archives, and teaching tools. The Jefferson Digital Commons allows researchers and interested readers anywhere in the world to learn about and keep up to date with Jefferson scholarship. This article has been accepted for inclusion in Department of Urology Faculty Papers by an authorized administrator of the Jefferson Digital Commons. For more information, please contact: JeffersonDigitalCommons@jefferson.edu. 


\title{
Urolithiasis Location and Size and the Association with Microhematuria and Stone-Related Symptoms
}

\author{
Costas D. Lallas, M.D., Xiaolong S. Liu, M.D., Allen N. Chiura, M.D., \\ Akhil K. Das, M.D., and Demetrius H. Bagley, M.D.
}

\begin{abstract}
Purpose: To conduct a study to assess the association between calculus location and size and the incidence of both microhematuria and symptoms of urolithiasis in a urology office environment.

Patients and Methods: After Institutional Review Board approval, a prospective study was conducted with data from 100 consecutive patients who presented to our office with documented urolithiasis. The location (caliceal, pelvic, or ureteral) and size ( $<$ or $\geq 8 \mathrm{~mm}$ ) of each calculus was determined from available diagnostic radiographs. The incidence of microhematuria was ascertained via a urine dipstick and microscopic examination. The presence of any symptoms associated with urolithiasis, including pain, subjective fever or chills, or urinary urgency, was recorded.

Results: A total of 111 stones were found in the study population resulting in a $45.9 \%$ incidence of microhematuria. In patients with renal pelvic and ureteral stones, $67.6 \%$ demonstrated microhematuria vs $36.4 \%$ with caliceal stones, $P=0.0035$. For stones $\geq 8 \mathrm{~mm}, 62.5 \%$ were positive for microhematuria $v s 29.1 \%$ of stones $<8 \mathrm{~mm}$, $P=0.0006$. Ureteral or renal pelvic stones caused the most symptoms $(70.6 \%)$ compared with caliceal stones $(16.9 \%), P=0.0001$. In those patients who reported pain associated with urolithiasis, $65.6 \%$ had concomitant microhematuria vs $36.8 \%$ in those without pain, $P=0.0097$.

Conclusions: Urinary calculus location and size are associated with the incidence of microhematuria and stonerelated symptoms. Pain related to urolithiasis may be a positive predictor for the presence of microhematuria.
\end{abstract}

\section{Introduction}

$\mathbf{T}$ HE PREVALENCE OF urinary lithiasis has increased in the United States during the past three decades. ${ }^{1,2}$ Approximately $10 \%$ to $15 \%$ of the US population will have an episode of urolithiasis in their lifetime with recurrence rates as high as $50 \% .^{3}$ Epidemiologic studies have shown that whites are almost three times more likely to have urolithiasis compared with blacks and men affected more often compared with women. ${ }^{4,5}$ Other risk factors include a positive family history and a variety of endocrine or metabolic abnormalities. ${ }^{6}$

Unfortunately, the occurrence of urinary lithiasis is not always a benign entity. Most commonly, these stone episodes can cause renal colic resulting in flank or abdominal pain, urinary urgency or frequency, fevers or chills, nausea or vomiting, and gross or microscopic hematuria. 7,8 The incidence of microhematuria and urolithiasis has been estimated to be as high as $90 \%$ in the literature and has been theorized to be present when the patient's pain is at its maximum intensity, diminishing to undetectable levels as the pain sub- sides. ${ }^{9,10}$ The absence of hematuria, however, does not always exclude the possibility of urinary lithiasis. Approximately $10 \%$ of patients may have a negative urine dipstick or microscopic examination on initial presentation. ${ }^{11}$ Nevertheless, urolithiasis remains a consistent finding in those undergoing workup for asymptomatic microhematuria and can be the representing etiology in as many as $16.5 \%$ of cases. $^{12}$

Previous studies have attempted to stratify the incidence of urinary lithiasis resulting in asymptomatic microhematuria to differentiate a more significant stone from ones of less consequence (eg, ureteral stone $v$ s a renal or vesical stone) and have suggested a possible association between calculus location and the presence of microscopic hematuria. ${ }^{13,14}$

The majority of the studies evaluating the incidence of microhematuria and urolithiasis have been limited to the acute-care setting-specifically patients presenting for evaluation in hospital emergency departments. ${ }^{15-18}$ Our objective was to perform a study to examine the incidence of microhematuria and urolithiasis-related symptoms and determine

Department of Urology, Thomas Jefferson University, Philadelphia, Pennsylvania. 
their possible association with the location and size of urinary calculi in an ambulatory office environment.

\section{Patients and Methods}

After Institutional Review Board approval, a prospective study was conducted by collecting data from 100 consecutive patients (63 males and 37 females) who were evaluated at our institution's urology office who had received a diagnosis of urolithiasis within a 6-month period. All patients had a documented diagnosis of a urinary calculus via radiographic imaging (eg, ultrasonography, radiography of the kidneys, ureters, and bladder, urography, or CT). Patients who had a documented urinary tract infection by a positive urinalysis and urine culture or those who were actively menstruating were excluded from the study.

The size and location of each calculus was determined using both information from the radiology report as well as evaluation by the office urologist with available imaging from each patient's respective diagnostic radiographs. Stone size was categorized as either $<8 \mathrm{~mm}$ or $\geq 8 \mathrm{~mm}$ (cutoff represented the median stone size). The location of all urinary stones was documented as renal calix (upper, mid, lower, or unspecified), renal pelvis/ureteropelvic junction (UPJ), or within the ureter (proximal or distal). Proximal ureteral stones were those located proximal to the sacroiliac joint, and distal ureteral stones were defined to be distal to this landmark. None of the calculi were located in a diverticulum or within the bladder.

Patients were interviewed with regard to their symptomsspecifically, flank or abdominal pain, subjective fevers or chills, or urinary urgency. Symptoms were recorded as either present or absent, depending on patient response. The presence of any of the aforementioned symptoms categorized the patient as positive for having stone-related symptomatology.

One urine specimen was obtained from each patient during the office visit. Each specimen was tested for blood using Chemistrip 10 strips $^{\mathrm{TM}}$ (Boehringer Mannheim Corporation, Indianapolis, IN). A color grading system of "negative, trace, $1+, 2+, 3+, 4+$ " was given with the product. "Trace" and greater dipstick reactions were considered positive for microhematuria. In addition, approximately $5 \mathrm{~mL}$ of each urine specimen was centrifuged at $2000 \mathrm{rpms}$ for 5 minutes. One drop of the resuspended pellet from the centrifuged urine was placed on a slide with a coverslip and examined under a microscope by a single urologist in the office. Between 10 and 15 high-power fields (at a magnification of $40 \times$ ) were inspected for each sample. The maximum number of red blood cells seen per high power field was noted. A patient was considered to be positive for microhematuria if more than three red blood cells per high power field were identified.

One physician interpreted both the urine dipstick and microscopic findings in all study patients. Those patients with multiple stones were categorized as having microhematuria in more than one stone category pending a positive result on urine dipstick or microscopy. In addition, the negative microhematuria rate, defined as a calculus negative for blood by either urine dipstick or microscopy, was also calculated.

Any patient with microscopic hematuria subsequently underwent a standard urologic hematuria evaluation, including a contrasted upper tract imaging study, urine cytol- ogy, and cystoscopy to rule out any associated pathology other than urolithiasis.

Statistical analysis was performed using the Fisher exact test with a $P$ value $<0.05$ demonstrating statistical significance (GraphPad Prism Version 5.03, GraphPad Software, La Jolla, CA).

\section{Results}

There were a total of 111 stones detected in the study population. The location and size distribution of each stone is shown in Table 1 . In all, $69.4 \%$ of the stones were located in the renal caliceal system and $30.6 \%$ in either the renal pelvis/UPJ or ureter. The median stone size was $8 \mathrm{~mm}$.

After examination by urine dipstick, the overall incidence of microhematuria was $45.9 \%$ compared with $37.8 \%$ by urine microscopy (Table 2). Stones in the renal pelvis/UPJ had the highest incidence of microhematuria at $82.4 \%$ by urine dipstick vs $52.9 \%$ of ureteral stones and $36.4 \%$ of caliceal stones. Comparable results are seen with microscopic analysis, because the rate of microhematuria was $76.5 \%, 52.9 \%$, and $26 \%$ for renal pelvis/UPJ, ureteral, and caliceal stones, respectively. In total, $67.6 \%$ of UPJ and ureteral calculi were dipstick positive compared with $36.4 \%$ of all caliceal calculi, $P=0.0035$. By microscopic analysis, $64.7 \%$ of UPJ and ureteral calculi were positive for microhematuria vs $26 \%$ of caliceal calculi, $P=0.0002$. The corresponding negative microhematuria rate for renal pelvis/UPJ, ureteral, and caliceal stones is also reported $(17.6 \%, 47.1 \%$, and $63.6 \%$, respectively), with the highest percentage of negative stones located in a midcalix $(81.8 \%)$.

The incidence of stone-related symptoms in relation to the location of urinary calculi is shown in Table 2. In total, 33.3\% of all stones contributed to patient symptoms relating to their urolithiasis. Overall, $70.6 \%$ of ureteral and renal pelvic stones resulted in the presence of one or more stone-related symptoms compared with $16.9 \%$ of all caliceal stones, $P=0.0001$. Proximal ureteral stones caused the highest rate of symptoms $(100 \%)$, while no symptoms were reported secondary to the presence of midcaliceal stones.

Urolithiasis size and the incidence of microhematuria are shown in Table 3. For stones $\geq 8 \mathrm{~mm}$, the incidence of microhematuria via dipstick analysis was $62.5 \%$ compared with $29.1 \%$ for stones $<8 \mathrm{~mm}, P=0.0006$. By urine microscopy $51.8 \%$ vs $23.6 \%$ were positive for microhematuria in stones $\geq 8 \mathrm{~mm}$ and $<8 \mathrm{~mm}$, respectively, $P=0.0032$. The corresponding negative microhematuria rates were $70.9 \%$ for

Table 1. Distribution of Urolithiasis Based on Size

\begin{tabular}{lrr}
\hline Location & $<8 \mathrm{~mm}$ & $\geq 8 \mathrm{~mm}$ \\
\hline Calix & & \\
Upper & 7 & 5 \\
Mid & 3 & 8 \\
Lower & 26 & 18 \\
$\quad$ Unspecified & 5 & 5 \\
RP/UPJ & 0 & 17 \\
Ureter & & \\
$\quad$ Proximal & 4 & 0 \\
$\quad$ Distal & 10 & 3
\end{tabular}

$\mathrm{RP}=$ renal pelvis; $\mathrm{UPJ}=$ ureteropelvic junction . 
Table 2. Association Between Calculus location and Incidence of Microhematuria and Stone-Related Symptoms

\begin{tabular}{lcccc}
\hline Calculus location & $(+)$ Hematuria dipstick & (+) Hematuria micro & $(-)$ Hematuria rate & $(+)$ Symptoms \\
\hline Calix & & & & \\
$\quad$ Upper & $4 / 12(33.3 \%)$ & $2 / 12(16.7 \%)$ & $8 / 12(66.7 \%)$ & $3 / 12(25 \%)$ \\
$\quad$ Mid & $2 / 11(18.2 \%)$ & $2 / 11(18.2 \%)$ & $9 / 11(81.8 \%)$ & $0 / 11(0 \%)$ \\
Lower & $19 / 44(43.2 \%)$ & $13 / 44(29.5 \%)$ & $25 / 44(56.8 \%)$ & $9 / 44(20.5 \%)$ \\
$\quad$ Unspecified & $3 / 10(30 \%)$ & $3 / 10(30 \%)$ & $7 / 10(70 \%)$ & $1 / 10(10 \%)$ \\
RP/UPJ & $14 / 17(82.4 \%)$ & $13 / 17(76.5 \%)$ & $3 / 17(17.6 \%)$ & $10 / 17(58.8 \%)$ \\
Ureter & $1 / 4(25 \%)$ & $1 / 4(25 \%)$ & $3 / 4(75 \%)$ & $4 / 4(100 \%)$ \\
$\quad$ Proximal & $8 / 13(61.5 \%)$ & $8 / 13(61.5 \%)$ & $5 / 13(38.5 \%)$ & $10 / 13(76.9 \%)$ \\
$\quad$ Distal & $51 / 111(45.9 \%)$ & $42 / 111(37.8 \%)$ & $60 / 111(54.1 \%)$ & $37 / 111(33.3 \%)$ \\
Overall & 0.0035 & 0.0002 & 0.0035 & 0.0001 \\
$P$ value & & &
\end{tabular}

${ }^{+} P$ value calculated by comparing all ureteral/UPJ/RP stones $v s$ all caliceal stones.

$\mathrm{RP}=$ renal pelvis; $\mathrm{UPJ}=$ ureteropelvic junction.

stones $<8 \mathrm{~mm}$ and $37.5 \%$ for those $\geq 8 \mathrm{~mm}$. With regard to patient symptomatology, $37.5 \%$ of stones $\geq 8 \mathrm{~mm}$ resulted in the presence of patient symptoms compared with $29.1 \%$ of stones $<8 \mathrm{~mm}, P=0.4219$ (Table 3 ).

In patients who specifically reported the presence of flank or abdominal pain, $65.6 \%$ were positive for microhematuria by urine dipstick compared with $36.8 \%$ for those without pain, $P=0.0097$ (Table 4). Of patients with urolithiasis-related pain, $56.3 \%$ had a positive microscopic analysis compared with $29.4 \%$ of patients without pain, $P=0.0147$. The corresponding negative microhematuria rates were $34.4 \%$ and $63.2 \%$ for those with and without pain, respectively.

\section{Discussion}

Urinary lithiasis has been an escalating medical problem with a yearly incidence of about $5 \%$ to $10 \%$ in North America and Europe. ${ }^{19,20}$ True incidence is difficult to quantify, because most patients with asymptomatic stones may never present for evaluation to the hospital or ambulatory setting.

The hallmark signs of an urolithiasis episode, including renal colic, urinary urgency or frequency, fevers/chills, and hematuria, account for more than half a million visits to the emergency department yearly. ${ }^{11}$ To our knowledge, however, the incidence of these findings has not been reported in the nonacute setting. In our study of 100 consecutive office patients, we found that the overall majority of stones diagnosed are asymptomatic (66.6\%). The location of urinary lithiasis, however, was associated with the presence of patient symptoms when they did occur. Those stones located in renal calices were the least symptomatic $(16.9 \%)$ when compared with renal pelvic $(58.8 \%)$ or ureteral stones $(82.4 \%)$. These findings are consistent with those reported by Elton and associates ${ }^{21}$ who found unilateral flank pain to be the predominating symptom in $89 \%$ of 206 emergency department patients with a diagnosis of ureteral calculus.

Physiologic principles support these findings, because ureteral calculi can result in urinary obstruction leading to severe renal colic secondary to renal capsular expansion and ureteral distention. ${ }^{16}$ In comparison, caliceal stones, assuming they do not migrate into the renal pelvis or ureter, should be the least symptomatic based on location and anatomy. There was a trend toward an increase in patient symptoms in association with larger stone size, but this difference did not reach statistical significance. In theory, larger stones in the ureter may result in higher degrees of obstruction and possibly lead to more pain. Future studies should stratify stone location based on size to determine if this perceived association exists with patient symptomatology.

Our results also indicate that the incidence of microhematuria by both urine dipstick and microscopic examination is associated with both calculus location and size. Stones located in the renal pelvis/UPJ and ureter were approximately twice as likely to cause microhematuria compared with those in the renal caliceal system. The exact mechanism of ureteral or UPJ stones leading to a higher incidence of microhematuria is difficult to elucidate, although the increased possibility of urothelial irritation or local inflammation is certainly related, and is corroborated by our stone location and patient symptom findings.

With regard to size, stones $\geq 8 \mathrm{~mm}$ were more than twice as likely to be associated with microhematuria as those $<8 \mathrm{~mm}$ by either urine dipstick or microscopic examination. This finding is particularly interesting given results from previous studies using $6 \mathrm{~mm}$ as a cutoff value that reported no difference in the rate of microhematuria based on calculus size alone. ${ }^{18}$

Our overall microhematuria rate was $45.9 \%$, which is significantly lower than the $91 \%$ reported by Li and colleagues ${ }^{11}$ in

Table 3. Association Between Calculus Size and Incidence of Hematuria and Related Symptoms

\begin{tabular}{lcccc}
\hline Calculus size & $(+)$ Hematuria dipstick & $(+)$ Hematuria micro & $(-)$ Hematuria rate & $(+)$ Symptoms \\
\hline$<8 \mathrm{~mm}$ & $16 / 55(29.1 \%)$ & $13 / 55(23.6 \%)$ & $39 / 55(70.9 \%)$ & $16 / 55(29.1 \%)$ \\
$\geq 8 \mathrm{~mm}$ & $35 / 56(62.5 \%)$ & $29 / 56(51.8 \%)$ & $21 / 56(37.5 \%)$ & $21 / 56(37.5 \%)$ \\
$P$ value & 0.0006 & 0.0032 & 0.0006 & 0.4219 \\
\hline
\end{tabular}


Table 4. Association Between Hematuria and Pain

\begin{tabular}{lccc}
\hline Pain & $\begin{array}{c}\text { (+) Hematuria } \\
\text { dipstick }\end{array}$ & $\begin{array}{c}(+) \text { Hematuria } \\
\text { micro }\end{array}$ & $\begin{array}{c}(-) \text { Hematuria } \\
\text { rate }\end{array}$ \\
\hline Present & $21 / 32(65.6 \%)$ & $18 / 32(56.3 \%)$ & $11 / 32(34.4 \%)$ \\
Absent & $25 / 68(36.8 \%)$ & $20 / 68(29.4 \%)$ & $43 / 68(63.2 \%)$ \\
$P$ value & 0.0097 & 0.0147 & 0.0097 \\
\hline
\end{tabular}

their series of 159,000 patients. Their study, however, was conducted as a retrospective review of patients who presented in the acute environment (ie, the emergency department) for evaluation of urolithiasis with the great majority of their stones $(76 \%)$ located at the ureterovesical junction or in the ureter, which may have lead to their higher overall rate of microhematuria. In comparison, $61.5 \%$ of our patients with distal ureteral stones were positive for microhematuria, which more closely resembles the results from their study population.

We report the incidence of microhematuria with both urine dipstick as well as microscopic examination in this study. Neither test is without its inherent flaws. False-positive urine dipstick results can occur secondary to hemoglobinuria, myoglobinuria, or contaminants such as hypochlorite or povidone-iodine. ${ }^{22}$ Red cell hemolysis, interobserver variability, and poor reliability can lead to false negative or positive results using urine microscopy alone. ${ }^{23,24}$ Our results note a higher incidence of microhematuria using urine dipstick when compared with microscopy. Other authors, however, have reported on the reliability of urine dipstick analysis for microhematuria, stating that a negative dipstick followed by a positive microscopic examination only added $2 \%$ to the diagnostic accuracy of urine dipstick in a study of 609 patients with urolithiasis. ${ }^{18}$ It is important to note that both urine dipstick and microscopy yielded statistically significant results in our study, indicating that the association between the incidence of microhematuria and stone location and size exists regardless of test method interpreted.

The presence of pain may be an independent predictor of microhematuria. Patient report of pain was associated with a statistically significant higher incidence of microhematuria by either urine dipstick $(65.6 \%)$ or microscopic examination $(56.3 \%)$. Although a direct link between pain and microhematuria is difficult to surmise, we again postulate that stones located in the ureter or UPJ may lead to more urothelial irritation and thus higher rates of microhematuria in this population.

Our absolute rates of microhematuria and patient reported symptoms are lower than those reported in the literature. Patients in our study were evaluated in the ambulatory office, a nonemergent setting, which inherently drives these values lower when compared with other studies based on findings during acute stone episodes. Thus, differences in the incidence of microhematuria and stone-related symptoms may have been more apparent given our patient population. In addition, specific urine dipstick results (ie, the number of patients with trace, 1+, 2+, 3+, 4+) and long-term follow-up data of asymptomatic microhematuria patients were not available for analysis. It would be interesting to investigate this in future studies and determine if the amount of microhematuria was associated with stone location or size or the occurrence of future symptomatic episodes in those who were initially asymptomatic.

\section{Conclusion}

In the ambulatory setting, microhematuria is not a universal finding in patients with urinary calculi and varies with stone location and size. The presence of pain may be an independent predictor of microhematuria in these patients with urolithiasis.

\section{Disclosure Statement}

No competing financial interests exist.

\section{References}

1. Stamatelou KK, Francis ME, Jones CA, et al. Time trends in reported prevalence of kidney stones in the United States: 1976-1994. Kidney Int 2003;63:1817-1823.

2. Johnson CM, Wilson DM, O'Fallon WM, et al. Renal stone epidemiology: A 25-year study in Rochester, Minnesota. Kidney Int 1979;16:624-631.

3. Sierakowski R, Finlayson B, Landes RR, et al. The frequency of urolithiasis in hospital discharge diagnoses in the United States. Invest Urol 1978;15:438-441.

4. Soucie JM, Thun MJ, Coates RJ, et al. Demographic and geographic variability of kidney stones in the United States. Kidney Int 1994;46:893-899.

5. Sarmina I, Spimak JP, Resnick MI. Urinary lithiasis in the black population: An epidemiological study and review of the literature. J Urol 1987;138:14-17.

6. Teichman JM. Clinical practice. Acute renal colic from ureteral calculus. N Engl J Med 2004;350:684-693.

7. Moe OW. Kidney stones: Pathophysiology and medical management. Lancet 2006;367:333-344.

8. Hall PM. Nephrolithiasis: Treatment, causes, and prevention. Clev Clin J Med 2009;76:583-591.

9. Rhea JT, Deluca SA, Toombs BD. Evaluation of a sequence of diagnostic tests using the workup of ureteral stone as a model. Med Care 1982;20:843-848.

10. Dunn PM, Keller RT, Jones SR. The absence of hematuria in patients with symptomatic urinary tract stones. West J Med 1985;142:717-719.

11. Li J, Kennedy D, Levine M, et al. Absent hematuria and expensive computerized tomography: Case characteristics of emergency urolithiasis. J Urol 2001;165:782-784.

12. Bagley DH. Hematuria in the adult. In: Coe FL, Favus MJ, Pak CY, Parks JH, Preminger GM, eds. Kidney Stones: Medical and Surgical Management. Philadelphia: Lippincott-Raven Publishers, 1996, pp 521-528.

13. Golin AL, Howard RS. Asymptomatic microscopic hematuria. I Urol 1980;124:389-391.

14. Carson CC III, Segura JW, Greene LF. Clinical importance of microhematuria. IAMA 1979;241:149-150.

15. Stewart DP, Kowalski R, Wong P, Krome R. Microscopic hematuria and calculus-related ureteral obstruction. J Emerg Med 1990;8:693-695.

16. Manthey DE, Teichman J. Nephrolithiasis. Emerg Med Clin North Am 2001;19:633-654.

17. Ooi SB, Kour NW, Mahadev A. Hematuria in the diagnosis of urinary calculi. Ann Acad Med Sinagpore 1998;27;210214.

18. Argyropoulos A, Farmakis A, Doumas K, et al. The presence of microscopic hematuria detected by urine dipstick test in the evaluation of patients with renal colic. Urol Res 2004;32: 294-297. 
19. Pak CY, Resnick MI, Preminger GM. Ethnic and geographic diversity of stone disease. Urology 1997;50:504-507.

20. Tiselius HG. Stone incidence and prevention. Braz J Urol 2000;26:452.

21. Elton TJ, Roth CS, Berquist TH, Silverstein MD. A clinical prediction rule for the diagnosis of ureteral calculi in emergency departments. J Gen Intern Med 1993;8:57-62.

22. Patel JV, Chambers CV, Gomella LG. Hematuria: Etiology and evaluation for the primary care physician. Can J Urol 2008 15(suppl 1):54-62.

23. Kiel DP, Moskowitz MA. The urinalysis: A critical appraisal. Med Clin North Am 1987;71:607-624.

24. Vaughn ED Jr, Wyker AW Jr. Effect of osmolality on the evaluation of microscopic hematuria. J Urol 1971;105:709-711.
Address correspondence to: Costas D. Lallas, M.D.

Department of Urology

Thomas Jefferson University

1025 Walnut Street, Suite 1112

Philadelphia, PA 19107

E-mail: costas.lallas@jefferson.edu

\section{Abbreviations Used}

$\mathrm{CT}=$ computed tomography

$\mathrm{UPJ}=$ ureteropelvic junction 
